\title{
UNIFORM STRUCTURE IN TOPOLOGICAL GROUPS
}

\author{
GERALD ITZKOWITZ
}

\begin{abstract}
A characterization is given of those locally compact groups having equivalent right and left uniform structures. It is shown that $\alpha$ compact locally compact groups have equivalent uniformities iff for each right uniformly discrete set $B$ such that card $(B) \leqslant \alpha$ and each neighborhood $U$ of $e, \cap_{x \in B} x U x^{-1}$ is a neighborhood of $e$. It is also shown that a group is not unimodular iff it contains an open $\sigma$-compact group which is not unimodular.
\end{abstract}

1. Introduction. In this paper we give a characterization of those locally compact topological groups that have equivalent right and left uniform structures. Our characterization is in terms of a notion called $\alpha$-compactness which is a generalization of $\sigma$-compactness in topological spaces.

Let $\alpha$ be a cardinal number. $G$ will denote a topological group.

Definition. A topological space $X$ is said to be $\alpha$-compact if $X$ can be written as a union of $\alpha$ compact sets and if $X$ cannot be written as a union of $\beta$ compact sets if $\beta<\alpha$.

The idea for using this approach to studying the right and left uniformities on a topological group came from two pieces of work. The first piece was a manuscript circulated among friends and colleagues concerning uniform structure in $\sigma$-compact locally compact topological groups. The second work is a paper [1] by Comfort, Itzkowitz, and Ross in which the problem of determining the size of closed subgroups of a topological group is solved in terms of $\alpha$-compactness in the case where a topological group is locally compact. We will show that in the case of locally compact $\alpha$-compact topological groups, that the problem of determining equivalent or inequivalent uniform structure is solvable in terms of discrete sets of cardinal $\alpha$ (if $\alpha$ is infinite).

We will follow definitions 4.11-4.13 in Hewitt and Ross [4] concerning right and left uniform structures on $G$. We will need the following observations concerning uniform structures for our paper.

1. The topology on $G$ defined by the left uniform structure consists of all the sets $\{x U: x \in G, U$ a neighborhood of $e\}$ and the topology on $G$ defined by the right uniform structure consists of the sets $\{U x: x \in G, U$ a neighborhood of $e$.

2. The right and left uniform structures are equivalent if for every

Received by the editors July 17, 1975 and, in revised form, October 22, 1975.

AMS (MOS) subject classifications (1970). Primary 22D05; Secondary 54E15, 54F65.

Key words and phrases. Equivalent uniform structure, $\alpha$-compact, unimodular.

- American Mathematical Society 1976 
neighborhood $U$ of $e$ there is a neighborhood $V$ of $e$ such that $x V x^{-1} \subset U$ for all $x \in G($ see $[4,4.14(\mathrm{~g})])$.

Notation. We will always denote the identity element of the group by $e$. $\operatorname{card}(A)$ will denote the cardinal number of $A$.

We will have occasion to use the following two important and classical theorems of topological groups.

[A] THEOREM [4, 4.9]. Let $G$ be a topological group, let $U$ be any neighborhood of $e$, and let $F$ be any compact subset of $G$. Then there is a neighborhood $V$ of $e$ such that $x V x^{-1} \subset U$ for all $x \in F$.

[B] THEOREM (KAKUTANI AND KodAIRA). Let $G$ be a $\sigma$-compact and locally compact topological group. Then for every countable family $\left\{U_{n}: n=1,2, \ldots\right\}$ of neighborhoods of $e$, there is a compact normal subgroup $N \subset G$ such that $N \subset \cap_{n=1}^{\infty} U_{n}$, and $G / N$ has a countable basis for its open sets.

Note. The theorem of Kakutani and Kodaira is proved for compactly generated groups in Hewitt and Ross [4, 8.7], and, for the more general case, in the original paper by Kakutani and Kodaira [6] and in Halmos [3]. Direct proofs may be found also in [2] and [5]. This theorem is the main tool of this paper and of [1] and [5].

\section{Inequivalent uniform structures in $\alpha$-compact groups.}

2.1. THEOREM. If $G$ is a locally compact and $\alpha$-compact topological group, and if $V$ is a neighborhood of $e$ then $\cap_{x \in G} x V x^{-1}$ is a neighborhood of $e$ if and only if for every set $A$ such that card $(A) \leqslant \alpha$ the set $\cap_{x \in A} x V x^{-1}$ is a neighborhood of $e$.

Proof. Let $H$ be an open $\sigma$-compact subgroup of $G$ and let $N$ be a compact normal subgroup of $H$ such that $H / N$ is a separable metric group (such an $N$ exists by Theorem [B]). It follows from 8.14(d) of [4] that $G / N$ is a metrizable space. Since $G / N$ is a continuous image of $G, G / N$ can be written as a union of $\alpha$ or fewer compact (metric) subsets. Thus $G / N$ has a dense subset $\tilde{J}$ where card $(\tilde{J}) \leqslant \alpha$.

For each $\tilde{x} \in \tilde{J}$ choose one distinguished element $x \in \varphi^{-1}(\tilde{x})$, where $\varphi: G \rightarrow G / N$ is the canonical map, and let

$$
J=\left\{x: x \text { is the distinguished element of } \varphi^{-1}(\tilde{x}), \tilde{x} \in \tilde{J}\right\} .
$$

Then obviously card $(J) \leqslant \alpha$ and so our hypothesis implies that $W$ $=\cap_{x \in J} x V x^{-1}$ is a neighborhood of $e \in G$. Thus since $N$ is compact we may apply Theorem [A] to conclude that

$$
\bigcap_{y \in N} y W y^{-1}=\bigcap_{y \in N} y\left(\bigcap_{x \in J} x V x^{-1}\right) y^{-1}=\bigcap_{z \in N J} z V z^{-1}
$$

is a neighborhood of $e$. Since $\tilde{J}$ is dense in $G / N$ it follows that $N J$ is dense in $G$. Thus we have shown the existence of a dense subset $D \subset G$ such that $W_{0}=\cap_{z \in D^{z} z^{-1}}$ is a neighborhood of $e$.

Choose now $y \in G$. Let $\left\{y_{\gamma}\right\}$ be a net in $D$ such that $y_{\gamma} \rightarrow y$. Then $W_{0} \subset y_{\gamma} V y_{\gamma}^{-1}$ for each $\gamma$, or equivalently $y_{\gamma}^{-1} W_{0} y_{\gamma} \subset V$ for each $\gamma$. Since $G$ is 
completely regular there is a neighborhood $U$ of $e$ such that $\bar{U} \subset V$. Obviously the above argument may be used to select a neighborhood $W_{1} \subset W_{0}$ such that $\cap_{z \in D^{2}} z^{-1} W_{1} z \subset U \subset \bar{U}$. Thus we see that $y_{\gamma}^{-1} w y_{\gamma} \rightarrow y^{-1} w y \in \bar{U}$ for each $w \in W_{1}$. Hence $y^{-1} W_{1} y \subset \bar{U} \subset V$. Since $y \in G$ was arbitrary and $W_{1}$ depends on $U$ it follows that $W_{1} \subset \cap_{y \in G} y V y^{-1}$ and thus $\cap_{y \in G} y V y^{-1}$ is a neighborhood of $e$.

The converse is obvious.

In what follows we will show that the study of equivalent uniformities can be reduced to the study of sets $A$ which have card $(A) \leqslant \alpha$ where $A$ is discrete.

Definition. The set $A \subset G$ is right-uniformly discrete if there exists a neighborhood $U$ of the identity in $G$ such that for all $x, y \in A$ where $x \neq y$ the relation $U x \cap U y=\varnothing$ holds.

2.2. Lemma. Let $G$ be locally compact and let $U$ be a neighborhood of $e \in G$ such that $\bar{U}$ is compact. Let $A \subset G$ be such that $\cap_{x \in A} x U x^{-1}$ is not a neighborhood of $e$. Then there is a right-uniformly discrete set $B \subset A$ such that $\cap_{x \in B} x U x^{-1}$ is not a neighborhood of $e$.

Proof. Let $\alpha=\operatorname{card}(A)$ and let $A=\left\{x_{\eta}: \eta<\alpha\right\}$ be a well ordering of $A$. We select the subset $B \subset A$ as follows.

The first element of $B$ is $x_{\eta_{0}}=x_{0}$. The second element of $B$ is $x_{\eta_{1}}$ where $x_{\eta_{1}}$ is the first element in the well ordering of $A$ such that $x_{\eta_{1}} \notin U x_{\eta_{0}}$. Using transfinite induction, let $J=\left\{\eta_{i}: \eta_{i}<\alpha\right\}$ be a set of ordinal numbers with the following properties:

(a) For each $j, x_{\eta_{j}} \notin \cup_{i<j} U x_{\eta_{i}}$.

(b) $\eta_{j}$ is the first ordinal greater than or equal to $\sup _{i<j}\left\{\eta_{i}\right\}$ such that (a) holds.

Then $B=\left\{x_{\eta}: \eta \in J\right\}$. We may observe that $A \subset U B$. We will show that $B$ is a right uniformly discrete set such that $U_{0}=\bigcap_{x \in B} x U x^{-1}$ is not a neighborhood of $e$.

Since $\bar{U}$ is compact Theorem [A] shows that if $U_{0}$ is a neighborhood of $e$ then $\cap_{x \in U} x U_{0} x^{-1}$ is a neighborhood of $e$. However,

$$
\bigcap_{x \in U} x U_{0} x^{-1}=\bigcap_{x \in U} x\left(\bigcap_{y \in B} y U y^{-1}\right) x^{-1}=\bigcap_{z \in U B} z U z^{-1} \subset \bigcap_{z \in A} z U z^{-1}
$$

since $A \subset U B$. This contradicts the fact that $\cap_{z \in A} z U z^{-1}$ is not a neighborhood of $e$.

To see that $B$ is right-uniformly discrete we let $W$ be a symmetric neighborhood of $e$ such that $W^{2} \subset U$. Then the collection $\{W x: x \in B\}$ is pairwise disjoint. To see this let $x_{\gamma}, x_{\beta} \in B$. Without loss of generality we may assume $\gamma<\beta$. Thus $x_{\beta} \notin U x_{\gamma}$ and therefore if $z \in W x_{\gamma} \cap W x_{\beta}$ we would have $z=w_{1} x_{\gamma}=w_{2} x_{\beta}$ so that $x_{\beta}=w_{2}^{-1} w_{1} x_{\gamma} \in W^{2} x_{\gamma} \subset U x_{\gamma}$, a contradiction.

2.3. TheOREM. Let $G$ be a locally compact and $\alpha$-compact group. Then $G$ has equivalent uniformities if and only if for each right-uniformly discrete set $B$ satisfying card $(B) \leqslant \alpha$ and each neighborhood $U$ of $e, \cap_{x \in B} x U x^{-1}$ is a neighborhood of $e$. 
3. Some further remarks and observations. In our constructions in the proofs of 2.1 and 2.2 it is not clear whether one needs sets $A$ with card $(A) \leqslant \alpha$. There is a possibility that even in the case where $G$ is $\alpha$-compact that the question of equivalent or inequivalent uniformities in the group may be settled just by the use of discrete sequences in the group. This would certainly be the case if every locally compact group with inequivalent uniform structures contains an open $\sigma$-compact subgroup with inequivalent uniform structures. While it is unlikely that this remark will be true, in general, it turns out that, in at least the case of $G$ being nonunimodular, it is true.

3.1. Theorem. Let $G$ be a locally compact topological group. Then $G$ is unimodular if and only if every open $\sigma$-compact subgroup of $G$ is unimodular.

Proof. If $G$ is not unimodular we have that $\Delta$, the modular function, and $1 / \Delta$ are unbounded on $G$. Thus we may select a sequence $\left\{x_{n}\right\} \subset G$ such that for each $n, \Delta\left(x_{n}\right)>n$. Let $U$ be a symmetric neighborhood of $e \in G$ such that $\bar{U}$ is compact. Then

$$
F_{n}=\bigcup_{j=1}^{\infty}\left[\bar{U} \cup\left\{x_{1}, x_{2}, \ldots, x_{n}\right\} \cup\left\{x_{1}^{-1}, x_{2}^{-1}, \ldots, x_{n}^{-1}\right\}\right]^{j}
$$

is a $\sigma$-compact open subgroup of $G$. Clearly $F=\cup_{n=1}^{\infty} F_{n}$ is an open $\sigma$ compact subgroup of $G$. Furthermore $\Delta_{F}=\left.\Delta\right|_{F}$ is not unimodular and is the modular function of $F$. The converse is easy.

\section{BIBLIOGRAPHY}

1. W. W. Comfort, G. L. Itzkowitz and K. A. Ross, Density character in topological groups (to appear).

2. F. P. Greenleaf and M. Moskowitz, Cyclic vectors for representations associated with positive definite measures: nonseparable groups, Pacific J. Math. 45 (1973), 165-186. MR 50 \# 2389.

3. P. R. Halmos, Measure theory, Van Nostrand, Princeton, N.J., 1950. MR 11, 504.

4. E. Hewitt and K. A. Ross, Abstract harmonic analysis. Vol. I: Structure of topological groups. Integration theory, group representations, Die Grundlehren der math. Wissenschaften, Band 115, Academic Press, New York; Springer-Verlag, Berlin, 1963. MR 28 \# 158.

5. G. L. Itzkowitz, Continuous measures, Baire category, and uniform continuity in topological groups, Pacific J. Math. 54 (1974), 115-125.

6. S. Kakutani and K. Kodaira, Uber das Haarshe Mass in der lokal bikompakten Gruppe, Proc. Imp. Acad. Tokyo 20 (1944), 444-450. MR 7, 279.

Department of Mathematics, Queens College (CUNY), Flushing, New York 11367 